\title{
Distributed Control of Chemical Process Networks
}

\author{
Michael J. Tippett Jie Bao \\ School of Chemical Engineering, The University of New South Wales, New South Wales 2052, Australia
}

\begin{abstract}
In this paper, we present a review of the current literature on distributed (or partially decentralized) control of chemical process networks. In particular, we focus on recent developments in distributed model predictive control, in the context of the specific challenges faced in the control of chemical process networks. The paper is concluded with some open problems and some possible future research directions in the area.
\end{abstract}

Keywords: Distributed process control, chemical process systems, process networks, plantwide control, distributed model predictive control.

\section{Introduction}

Modern chemical processing plants consist of process units arranged in a complex network. Due to safety, economic and environmental concerns, there has been an increased use of energy integration and material recycle to improve steady state efficiency and reduce waste. These large networks can lead to difficulties in plant-wide analysis and control design, as the resulting large-scale system may have complex nonlinear dynamics. These systems provide difficulties for traditional centralized and decentralized control techniques. The distributed (or networked) control paradigm is promising as it allows for simple controllers which can communicate and cooperate with one another to achieve improved control performance and operational efficiency whilst remaining transparent and scalable.

Modern chemical processing networks are often designed with steady state efficiencies and capital costs in mind, as such, they often suffer from operability issues ${ }^{[1]}$. Heat integration and material recycle can cause strong coupling between process units, this coupled with tighter design margins can improve steady state performance at the cost of reduced flexibility ${ }^{[2,3]}$. The design of plant-wide control systems for these systems is a difficult problem, with key challenges provided by: 1) Process systems exhibit strongly nonlinear behavior, this greatly increases the complexity of analysis and associated control design. 2) They are largescale systems with strong interaction effects, due to complex network topologies (e.g., due to material recycle and heat integration). The scale of the problem means that computationally efficient methods are required, whilst the strong interaction effects must be accounted for to ensure plant-wide stability. 3) There is often significant time-scale separation between, and within, unit processes, due to dif-

\footnotetext{
Survey Paper

Manuscript received December 1, 2014; accepted February 10, 2015

This work was supported by Australian Research Council (ARC) Discovery Project (No. DP130103330)

Recommended by Associate Editor Yi Cao

(C) Institute of Automation, Chinese Academy of Science and Springer-Verlag Berlin Heidelberg 2015
}

ferent physical and chemical processes. For example, mass transport and chemical reaction effects may happen at very different rates. Furthermore, interaction effects can induce time-scale separation even when not present in the isolated process units $^{[4]}$. 4) Process Models often have significant model-plant mismatch, as complex physical-chemical first principle models often need to be simplified for control design. Additionally, physical parameters may be difficult to measure (or be time varying), leading to modelling errors.

Distributed control, whereby local controllers make autonomous decisions based upon knowledge of their local process unit and communication signals from remote controllers, has recently gained significant attention in the literature due to its scalability for large-scale applications and its potential to account for interactions between subsystems. A recent survey on distributed model predictive control (MPC) can be found in [5]. A significant advantage of these approaches over traditional, decentralized control systems is that interactions between unit processes can be accounted for at the regulatory control layer. This is in contrast to decentralized approaches whereby interactions between unit processes may be treated as uncertainties transforming the problem into a robust control problem i.e., $[6,7]$. This kind of decentralized approach can lead to poor performance as the known interactions are treated as being unknown. In contrast, in a distributed control approach, the controller communication allows for beneficial interactions to be utilized to improve plant-wide performance.

Centralized multivariable control is an intuitive approach. However, there are severe limitations in its application to large-scale systems, the most obvious of which is computing power, although this is neither the most fundamental nor worrisome issue ${ }^{1}$. In [8], an overview of centralized control is given. Some key issues were identified, and are as follows: requirement of a multivariable dynamic model, lack of fault tolerance, difficulties in tuning and poor transparency. An eloquent discourse on the practi-

\footnotetext{
${ }^{1}$ Although, this is very important in approaches requiring realtime optimization such as centralized MPC.
} 
cal limitations of centralized optimal control is presented in [9]. There are advantages of centralized control, with the main advantage being the control of strongly interacting processes. As pointed out in [10], however, these advantages may not be enough to justify the implementation of a centralized structure, where cascading feedback loops (decentralized control) may be effective.

Distributed control offers a solution to these pitfalls, of particular importance for nonlinear control design is modelling and computational burden. In the case of process networks, it is far easier to take a "divide and conquer" type approach of modelling individual unit processes and their interactions, than to develop a monolithic dynamic plant model due to their strong nonlinear nature. This results in a set of models that are naturally suited to distributed control design. Furthermore, the distribution and parallelization of the computational burden amongst a set of distributed controllers has demonstrated computational savings ${ }^{[11]}$.

In this paper, we present a review of the current literature on distributed (or partially decentralized) control of chemical process networks. In particular, we focus on recent developments in distributed model predictive control and how stability and performance objectives can be enforced. In contrast to some existing reviews (e.g., $[5,12]$ ), we also review some approaches to distributed model predictive control (such as dissipativity and passivity based methods) which have not been covered previously.

\section{Plant-wide process control}

Modern chemical processes consist of many units interconnected with one another, often through quite complicated network structures ${ }^{[13]}$. In particular, mass recycle and heat integration are commonly used in chemical process plants to improve steady state efficiency. However, these network structures represent positive feedback loops in the system, which are well known to be deleterious to control performance. Control of such processes is characterized by their scale, strong interactions and differing dynamics (possibly on different time-scales) of each process unit $^{[8]}$. In practice, the conventional approach is to control such systems by hierarchical control systems operating on different time-scales ${ }^{[10]}$. These range from the regulatory control layer interfacing directly with the process (seconds), supervisory control possibly including predictive control (minutes), local optimization (hours), plant-wide optimization (days) and scheduling (weeks). At each level, the controllers may communicate with one another (horizontal interaction) to account for interactions between process variables. In addition to these interactions, controllers in different levels interact with one another, e.g., upper level controllers set the setpoints for lower level controllers (vertical interaction). This is shown diagrammatically in Fig. 1.

There is a vast literature surrounding the regulatory and supervisory control layers. In particular, many recent works have focused on distributed (or partially decentralized) con- trol, whereby controllers on these levels communicate with one another to improve performance. This review takes a particular focus on distributed model predictive control, and available methods for ensuring stability and performance requirements.

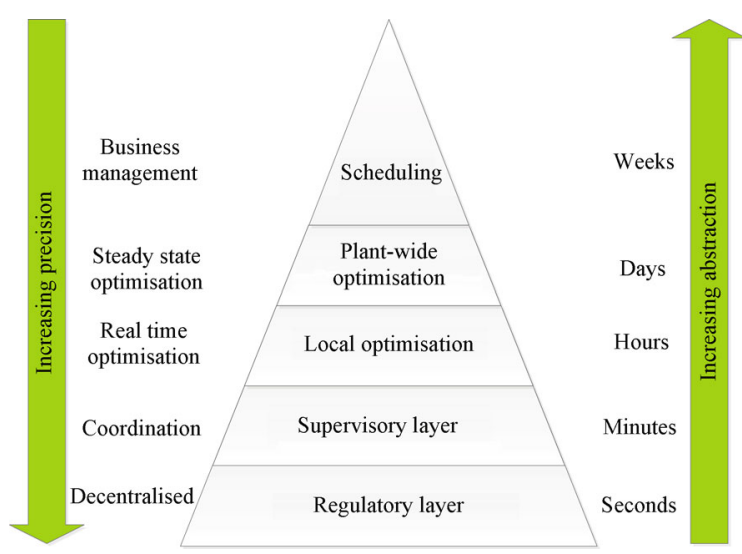

Fig. 1 Plant-wide control hierarchy (adapted from [14])

The broader question of how to integrate this with the other control layers in Fig. 1, as well as control structure selection (Which variables should be measured? Which should be manipulated?) are issues in the broader subject of plant-wide control ${ }^{[10]}$. Clearly, one key issue of distributed process control is to handle interactions between process units, particularly due to materials recycling and energy integration. Important considerations in this context are the underlying physical laws (e.g., conservation of mass, the laws of thermodynamics) which describe the process network, as they impose limitations on the available degrees of freedom. For example, it is well known that it is not possible to independently control both liquid inventory and flow rate, as they are linked. In the context of systems with recycle, improper choice of the controlled variable can lead to the "snowball effect" ${ }^{[15]}$, whereby small disturbances can have a large effect on recycle flow rates. The reader is directed to [13] for a further discussion on the topic of plant-wide control.

A link between controllability analysis and control is provided by the idea of self-optimising control introduced by Skogestad ${ }^{[8,16]}$, whereby the control structure is designed so that it is optimal with respect to disturbances when the process is operating at the setpoint. An advantage of this approach is that it provides a systematic method of determining the optimal control structure. An approach to achieve this optimality was presented in [17] and further extended in [18], which facilitates multiobjective controllability analysis and the development of Pareto optimal solutions. In the latter work, developments are presented which allow for the sensitivity of the solutions to errors and disturbances to be derived. 


\section{Distributed process control}

As has been mentioned previously, distributed control refers to the case where a large-scale system is controlled by a series of local controllers, that are allowed to communicate with one another. This is shown diagrammatically in Fig. 2. This may be viewed as two interacting networks: a process network interacting via mass and energy flows, and a controller network interacting via information flows.

An interesting question in distributed control design is how to select the controller network structure. In some approaches, the structure is set by the design, e.g., the controller and process network topologies must coincide with one another in $[19,20]$, and the controller network has a full structure (i.e., each controller communicates with every other controller) in [21]. For approaches where the controller communication network topology may be chosen by the designer, the obvious question of "optimality" in controller communication structure is raised. One solution to the problem of determining the optimal controller network topology is presented in [22]. This is achieved in the context of the distributed linear quadratic (LQ) problem where the cost function is a function of the controller network topology. Interestingly, it is shown that adding communication links may be detrimental to performance by this measure in some cases. It may be difficult to reconcile this performance measure with other (more classical) ones.

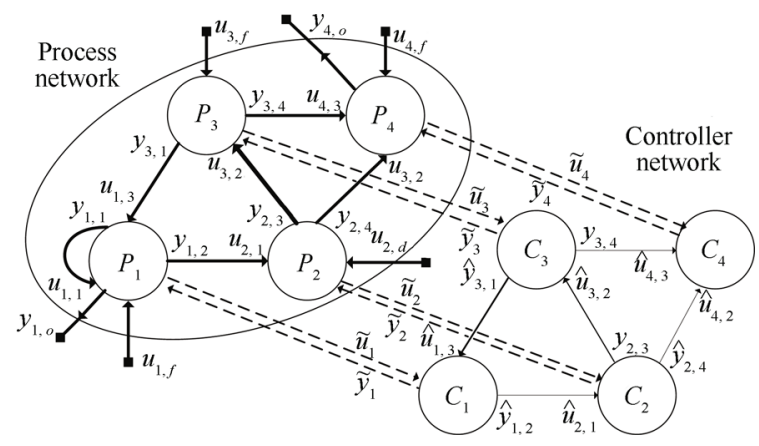

Fig. 2 Conceptual view of distributed control

Modern communication technologies offer a wide variety of methods by which the controller communication can be implemented, each of which have advantages and disadvantages. Recently, there has been considerable interest in these issues (under the name of networked control systems), e.g., the issue of uncertain delay resulting from ethernetbased internet protocols is studied, and an approach to robust control design is presented ${ }^{[23]}$. The general framework developed therein enjoys the advantage of being applicable to systems with nonlinearities in the communication network, and facilitates the allowable bounds on the delay and nonlinearities to be determined through convex optimization. An approach to networked model predictive control of chemical plants is presented in [24] using a two tier architecture, which allows for an upgrade of existing control infrastructure with modern wireless communication. From a control theory perspective, what is important is the impli- cations of the implementation of the controller communication network (e.g., the resulting time delays, presence and nature of noises). As such, in the remainder, we will focus on the structure of information flow between controllers, rather than how it is transmitted.

\subsection{Distributed model predictive control}

Distributed model predictive control (DMPC) has been an area of intensive research for both the process control and broader control theoretic community in recent years ${ }^{2}$. DMPC has three key advantages: ease of tuning, potentially optimal solution and effective constraint handling. The latter is of particular interest, as classical control techniques tend to handle constraints poorly or in an unsystematic manner. A key difficulty in applying MPC to large-scale systems has historically been the computational effort required to perform the online optimization. This has been one of the key driving forces for the development of distributed MPC techniques. Some existing reviews of recent developments and architectures of MPC can be found in $[12,26]$. DMPC can be broadly categorized into cooperative and non-cooperative approaches. In the former, the individual controllers act together to optimize a global cost function by manipulating their local process variables. Whilst in the latter, each controller optimizes its own local cost function by taking the actions of other controllers into account (although they do not take into account the effect of their own actions on other controllers' cost functions).

\subsubsection{Non-cooperative DMPC}

In non-cooperative DMPC, the individual controllers optimize their own local cost functions by taking the actions of the other controllers into account. An advantage of this approach is that the resulting algorithms are often simpler, and may require fewer communication channels and iterations between controllers. One such approach is presented in $[27,28]$ for linear systems, this approach is a general one which may be applied to dynamically coupled systems (such as process networks) and non-dynamically coupled systems (such as vehicle formations). An advantage of this approach is that each controller only needs to know the reference and state trajectories of their neighbors, and no further state information from the rest of the plant or models of the dynamics of other processes is required. A key requirement of this approach is then that each controller ensures that the closed-loop trajectory of its local process remains within a certain distance of the reference signal. Some implementation issues of this algorithm, such as selection of tuning parameters, initialization and disturbance handling, are studied in [29].

A similar requirement (called a consistency constraint) is placed on the algorithm developed for dynamically coupled nonlinear systems in [30]. An important assumption in

${ }^{2}$ Decentralized MPC, due to its comparative simplicity and similarity to existing industrial practice, has been of interest for a longer period, with rigorous treatments of the stability of decentralized MPC strategies for dynamically coupled systems appearing from at least the 1990s, e.g., [25]. 
this approach is that the input constraints on the individual controllers are decoupled. This is a realistic assumption in process control applications in which the input constraints are most often due to actuator limitations, and thus independent of the rest of the process network. It is shown in this paper that the computational complexity (in terms of calculations required) is significantly smaller than that in the centralized case, and even more so if the parallelization of computation is taken into account. However, there is an increase in the amount of data traffic that the communication system must handle, with modern communication technology this is likely to be more than offset by the decreased computational load, however.

In the above approaches, the controllers communicate in an iterative fashion, whereby the controllers iteratively optimize their local objectives and communicate with other controllers until some halting condition is met (e.g., convergence of solutions, maximum number of iterations). However, another approach is for the controllers to communicate sequentially, in which case there is a hierarchy of controllers which compute their optimal solution and communicate it to the next controller in line each sampling instance. This approach can yield a substantial decrease in the required computation and communication, as well as making proof of convergence of each controller easier. It is noted in [31] that cycles in the process network, due to recycle and heat integration for example, complicate iterative DMPC applications. As such, iterative minimax optimization (whereby each controller carries out its optimization based on the worst case possible interactions from other process units) or sequential communications are suggested as possible solutions. However, iterative communication may come at the expense of decreased performance, especially in the final controller in the hierarchy, as it may have limited freedom to achieve its local performance objectives given the decisions of the previous controllers in line. As such, the choice of optimization hierarchy is important and should be driven by knowledge of the interactions between processes. One such approach which fits into this category is that presented in [32], where a hybrid linear and nonlinear DMPC approach is developed. This approach uses nonlinear model predictive controller (NMPC) to deal with strong nonlinearities occurring in certain process units (e.g., chemical reactors) and allows for simpler linear MPCs to be applied to the process units with weaker nonlinearities (e.g., distillation columns).

Another nonlinear DMPC approach which uses sequential communication is presented in [11], wherein the framework is general enough to allow for either sequential or iterative communication. This approach is one of a class of MPC algorithms called Lyapunov MPC, initially developed in $[33,34]$ for the control of single systems. In this approach, the amount by which the MPC decreases a Lyapunov function is compared to a known stabilizing controller. If the Lyapunor MPC decreases the Lyapunov function more than the known controller, the control action of the Lyapunov MPC is used, otherwise that of the known controller is applied. In this way, the MPC inherits the stability properties of the known controller. The motivation for using MPC in this approach is to improve the performance of the control system by using MPC, and thus achieving optimal constrained control. This is particularly important when economic considerations are considered, as economic MPC allows for these to be optimized explicitly at the supervisory and regulatory control layers in real-time rather than at higher levels in the plant-wide control system. Extensions of this approach to economic MPC have recently been reported in [35-37], the latter two of which focus on output feedback. In addition to this, there have been many developments of Lyapunov MPC in cooperative DMPC, which will be discussed in the following section.

As mentioned above, one method to decrease the amount on computation (and communication) required in noncooperative DMPC is to design local controllers such that they solve a local minimax problem based on the worst case interactions from other process units. In this approach, the interactions between process units are treated as unknown disturbances, as the predicted trajectories sent from one controller to another are assumed to be potentially inaccurate. As such, there are many parallels between this approach and robust MPC formulations, and optimization under uncertainty more generally ${ }^{[38-40]}$. The above approach has the advantage of allowing communication to be halted before each controller iterates to convergence, as in 41,42 . In these frameworks, it may be necessary for the local controllers to enforce bounds on their predicted state/output trajectories so as to ensure plant-wide stability. A disadvantage of this approach is that the assumption of worst case disturbances can lead to poor performance. Furthermore, if the feedback nature of the receding horizon framework is not taken into account, it can be difficult to ensure feasibility. The latter point may be addressed by designing feedback minimax approaches ${ }^{[39]}$, or as detailed in the excellent survey paper ${ }^{[43]}$.

The dissipativity-based DMPC approach for linear process networks presented in $[44,45]$ is also classified as a noncooperative strategy. In this approach, a dissipativity ensuring constraint is placed on each local controller. This, in turn with the dissipativity properties of the individual process dissipativity properties, and process and controller network topologies, is used to ensure stability and minimum performance bounds on the closed-loop plant-wide system. This can be seen as an extension of the approach presented in [46] to distributed control and more general forms of dissipativity. An advantage of dissipativity based approaches is that dissipativity may be used to effectively bound the input-output properties of the processes, allowing for interactions between processes to be accounted for. This also allows for a "trading" of stability between processes, as controllers may be designed to make use of stabilizing influences of certain process units on others to help stabilize other processes. A potential disadvantage of this is that each closed-loop may then not be stable in isolation, or when the controller communication network fails. To 
rectify this, an approach to ensure stability in these cases has been presented in [47]. Additional extensions to multirate control have also been developed ${ }^{[48]}$. As the stability results obtained from dissipativity-based analysis are sufficient only, there could be some reduction in performance due to the need to satisfy these conditions. On the the other hand, as dissipativity represents bounds on the system properties, it is well suited to describing uncertainties. Indeed, $H_{\infty}$ robust control may be cast in the framework of dissipativity. Thus, there is the possibility of extending these approaches to robust distributed MPC.

A key feature of this dissipativity-based DMPC approach is the use of quadratic difference forms (QdFs) as supply rates of the processes and controllers ${ }^{3}$. These forms allow for sharper stability results as compared to traditional supply rates. Futhermore, as they may be thought of as quadratic forms of a finite, receding, horizon of a system's inputs and outputs, they are well suited to the MPC framework. This approach can be seen as a special case of some of the dissipativity based distributed process control (DPC) approaches presented in Section 3.2.

\subsubsection{Cooperative DMPC}

In cooperative DMPC, the controllers manipulate their local variables in order to achieve a globally optimal solution, that is to optimize a global cost function. This allows for the possibly deleterious effects of one controller's actions on other controllers and therefore global performance to be taken into account. This is in contrast to the noncooperative approaches discussed above, where the effect of each controller's action on others is only considered so far as to maintain stability, or possibly a minimum performance level. As such, cooperative control facilitates the realization of the Pareto optimal solution, as compared to non-cooperative control where the Nash equilibrium is typically obtained.

As pointed out in [51], the exchange of predicted state trajectories and the modelling of interactions between systems alone is insufficient to ensure stability in DMPC applications due to competition between controllers. Additionally, Bawlings and Stewart ${ }^{[51]}$ presents some interesting analysis and discussion on methods by which the amount of controller communication may be decreased by considering the structure of the process network, and by modifying interaction models. Although it was observed that there is a tradeoff, in that strong interactions require a higher degree of communication.

Some additional works by the same group as the above $^{[21,52-54]}$. A key feature of these developments is the emphasis on coordination between controllers to achieve a Pareto optimal solution, and studying the stability properties assuming that the optimal solution is obtained. Additionally, there has been a focus on studying the properties (i.e., stability and feasibility) of suboptimal MPC. For example, cases where the controllers are not allowed to iteratively communicate until each controller solution converges.

${ }^{3}$ See $[49,50]$ and the references therein for a mathematical description of QdFs.
This is important in practice as there may be limitations to the computational and communication overhead which is allowable. A key result in this context is that the solution of the local controller optimization algorithms must be feasible and maintain closed-loop stability after an arbitrary number of iterations. This is in contrast to many other MPC algorithms where stability is only ensured if the controllers are allowed to iterate to completion and the optimal solution is found. The key technical developments for this analysis appear in [55] in the context of MPC design for isolated systems, or from another perspective, centralized MPC design.

An extension of the above results to tracking control for non-zero setpoints (in the case of linear systems) is presented in [56]. This DMPC is able to drive the process network to any feasible setpoint using a feasible state and input trajectory. The use of an invariant set for the tracking problem based on a centralized controller gives the approach a larger region of attraction than standard cooperative DMPC formulations. This comes at the computational cost of having to determine this invariant set based on a centralized solution to the problem. However, it should be noted that this is carried out offline, and thus does not affect the computational complexity of the online problem. The approach presented in this paper can be thought of as a two layer structure, where the DMPC system performs as described above, and the upper layer coordinates the dynamic models used in the DMPC (supervisory/regulatory control layer) and the static models used at higher levels of the plant-wide control hierarchy (possibly a real-time optimization layer).

A novel sensitivity based coordination approach to DMPC is presented in $[57,58]$. A key development in this work is the use of a linear approximation of the cost functions of neighboring controllers in each controller's cost function in order to coordinate the individual controllers. The essential idea being that these approximations convey gradient information about the neighboring controllers' cost functions. Whilst a condition ensuring the convergence of the algorithm is presented, the paper does not consider the stability of the plant-wide system, leaving this as a potential area of future research.

A useful framework for studying DMPC is game theory, as it is well suited to studying optimal decision making amongst multiple agents. Furthermore, it allows for communication and negotiation protocols to be designed so that the controllers can either achieve cooperative or noncooperative solutions. In addition to optimality, this framework also allows for the stability of the resulting equilibria to be studied. Two game theoretic approaches are presented in $[59,60]$, the former focused on the case where there are only two subsystems. In this case, the amount of communication can be exactly determined, and practical stability of the resulting system is ensured. The latter paper considers the more general case of an arbitrary number of subsystems. As it is assumed that each controller only has local state and model information, the controllers must negotiate 
in order to achieve a cooperative solution. The individual controllers propose solutions, then they agree on the best solution based on a novel negotiation protocol. However, the game theory based approaches may suffer from combinatorial explosion of possible strategies, and thus become impractical when the number of subsystems is large. Also, the Nash equilibrium reached by independent controllers can be far from the Pareto optimal solution.

As mentioned in the previous section, the recently developed Lyapunov MPC, provides a stability ensuring framework for cooperative nonlinear DMPC. Many developments in this area have been summarized in the recent monograph $^{[26]}$. This is a flexible framework which allows for flat and tiered controller structures ${ }^{[24,61]}$, as well as asynchronous sensor information ${ }^{[62]}$, and process networks with multiple time-scales ${ }^{[63,64]}$. Additionally, this approach has been extended to allow for disruptions (e.g., noise or data losses) in the controller communication, by implementing a system which determines if the information received by a controller is reliable or not ${ }^{[65]}$. A significant advantage of this approach is its flexibility to handle a multitude of situations, and issues encountered in practical applications. Additionally, it allows for economic DMPC, as presented in [66]. Wherein the closed-loop and computational performance is compared to that of conventional centralized MPC, with the conclusion that economic DMPC allows for improved performance with the same level of computational burden. A potential disadvantage of this approach is that the designer must have knowledge of a stabilizing controller (which respects any constraints), which may be difficult to determine a priori. However, as many chemical process networks can be stabilized (at least locally) by relatively simple controllers (multi-loop or cascaded PID for example), this may not be major drawback in practice.

Although DMPC has been demonstrated to have significant computational savings as compared to centralized control ${ }^{[61,67,68]}$, the computational complexity of DMPC (especially for nonlinear systems) is still of concern. It was shown in [69] that there is a tradeoff between the rate of convergence, level of communication and the distribution of computation. This has led to the development of several approaches which reduce the amount of communication required $^{[28,59,70]}$. Another approach to reduce the computational requirements of DMPC is to develop specialized optimization algorithms which take advantage of the structure of the optimization problem which occurs in DMPC. One such approach for linear DMPC is presented in [71], based on offline inversion of the Hessian matrix and a dualmode strategy which allows for the designer to decrease the computational time at the expense of global optimality.

There have also been some parallel quadratic programming algorithms which may be applied to linear DMPC. In [72], such an approach based on accelerated gradient methods was developed using a dual decomposition. In [73], an algorithm based on the proximal center decomposition method was developed. A projection free approach to parallel quadratic programming was presented in [74]. However, it should be noted that these algorithms were not developed with DMPC in mind. And as such they do not consider the similarity of the optimization problem between two sampling instants. Therefore, some additional improvements may be possible.

A very computationally efficient approach to linear MPC is explicit MPC, first developed in [75, 76]. However, to the best of our knowledge, there have not been attempts to extend this to DMPC, and it remains restricted to systems of small or intermediate size due to the size of the resulting look up table in large-scale applications.

\subsection{Dissipativity and passivity based DPC}

Dissipative system theory was first formalized ${ }^{[77,78]}$ as an extension of the concept of passive systems. Intuitively speaking, dissipative systems are those for which the increase in stored energy is bounded by the amount of energy supplied by the environment (here energy may refer to actual physical energy or an abstract energy-like quantity). This provides a useful framework for studying interconnected systems, such as process networks, as it is an input-output property. This allows for much of the complexity of the problem to be shifted to the interconnection relations, rather than studying centralized process models. A continuous time dynamical system with input, output and state $u, y$ and $x$ respectively, is said to be dissipative if there exists a function called the supply rate $s(u, y)$, and positive semi-definite function defined on the state, called the storage function $V(x(t))$ such that:

$$
V\left(x\left(t_{1}\right)\right)-V\left(x\left(t_{0}\right)\right) \leq \int_{t_{0}}^{t_{1}} s(u(t), y(t)) \mathrm{d} t
$$

for all times $t_{0}, t_{1} \geq 0^{[77]}$. This inequality is known as the dissipation inequality. The following $(Q, S, R)$-type of supply rate is commonly used:

$$
s(u(t), y(t))=y^{\mathrm{T}}(t) Q y(t)+2 y^{\mathrm{T}}(t) S u(t)+u^{\mathrm{T}}(t) R u(t) .
$$

In the special case that $\operatorname{dim}(y)=\operatorname{dim}(u)$ and the supply rate is $y^{\mathrm{T}}(t) u(t)$, the system is said to be passive; which in the case of linear time invariant systems is equivalent to positive realness. As stability conditions based on dissipativity theory are sufficient only (most approaches use variations of the results developed in the series of papers ${ }^{[79-81]}$ ), there may be a need to detune the resulting controllers so as to ensure that these conditions are satisfied. The advantage of dissipativity theory for large-scale systems is that the analysis and control design may be carried out using the relatively simple dissipativity properties rather than detailed models.

Developments over the last two decades have drawn relationships between the thermodynamic laws underlying chemical process systems and passivity/dissipativity, see [82-88] and the references therein. In general terms, the available work of process systems may be used to define a 
storage function for chemical process systems in a natural way. This facilitates and motivates the use of dissipative controllers for such systems. The key result of $[82,83,87]$ is that under certain conditions, chemical process networks are structurally passive due to the 1st and 2nd laws of thermodynamics. Thus, by using the well known passivity theorem, it can be shown that they can be stabilized by decentralized passive controllers, e.g. multi-loop PID control. However, it should be noted that this result says little about the level of performance that may be realized with this approach. As this is a structural property of process systems (i.e., independent of certain parameter values), this approach may be robust to variations or inaccuracies in the measurements of these parameters.

A natural extension of this is the dissipativity with supply rates of the form (1). Dissipativity based analysis for plant-wide stability and performance was developed for the linear systems case ${ }^{[89]}$ and for nonlinear plant-wide systems $^{[90,91]}$. Dissipativity based decentralized control approaches were investigated for linear systems, where the controller design was formulated as a linear matrix inequality (LMI) problem ${ }^{[92,93]}$. This approach was then extended to distributed control ${ }^{[94,95]}$. This was achieved by reformulating the distributed control problem as a decentralized control problem of closed-loop nodes in the process network which interact through mass/energy and information ports, i.e., a two-port network. The disadvantage of this is that there is some restriction on the topology of the controller communication network. A related approach, which uses dynamic supply rates as a more general form of dissipativity is presented ${ }^{[96,97]}$. An advantage of this approach is that the more general supply rates allow for sharper stability and minimum performance bounds as compared to $(Q, S, R)$ supply rates.

In $[19,98]$, an LMI based $H_{\infty}$ approach to distributed control for linear systems, interconnected by either periodic or infinite structures is presented. In this context, the $H_{\infty}$ control problem may be seen as a special case of dissipativity based control where the supply rates are of the form $-y^{\mathrm{T}}(t) y(t)+\gamma^{2} u^{\mathrm{T}}(t) u(t), \gamma>0$. In the latter paper, imperfect controller communication in the form of lossy communication and constant delays is also considered.

An approach to dissipativity based distributed control for nonlinear systems based on differential geometry is presented $^{[20]}$. The essence of the approach is to decompose the drift vector field of each process into dissipative and non-dissipative components and then design controllers to dominate the non-dissipative component. An attractive feature of this is that it provides a constructive procedure for finding the process storage functions. Furthermore, dissipative (stabilizing) dynamics of the processes are retained allowing for less control effort to be used.

\subsection{Graph theoretic DPC}

Graph theory provides a natural framework for representing large-scale systems, as it allows for the structure of the relationships between nodes (which may represent unit pro- cesses or individual process variables) to take center stage. This is important in the context of both process design and control, as it is often the relationship/interactions between subsystems which are the source of performance difficulties. It has been suggested that graph theoretic approaches allow for a more flexible design procedure as compared to geometric approaches, however, with the caveat that the approach is strongly influenced by the choice of state variables ${ }^{[99]}$. Graph theoretic techniques, such as reduction of the graph representing the process network, can be also used prior to the use of other techniques. For example, an approach of reducing the graph of the process network into a strongly connected graph is advocated prior to dissipativity based stability analysis ${ }^{[100]}$.

In $[22,101]$, a graph theoretic DPC approach is presented, in which a "communication cost" is applied in a modified linear quadratic regulator (LQR) framework to find an optimal controller network structure. An interesting result in this formulation is the existence of (and conditions for) "critical prices" where the addition of further communication links decreases plant-wide closed-loop performance.

Another approach to develop the optimal controller structure (for both stabilization and disturbance rejection problems) is presented for nonlinear process systems ${ }^{[102]}$. In this approach, the process network is represented as a directed graph to describe the relationship between different internal and external variables. An approach for robust distributed large-scale systems both with and without timedelay in the communication channels between controllers is presented $^{[103]}$. As with the above paper, the process network is represented as a directed graph, this is a natural way to represent chemical process networks as the mass flows between process units are almost exclusively unidirectional in nature.

\subsection{Distributed state estimation}

An important implementation issue in modern control design is state estimation. In the case of distributed control, this issue is compounded by the need for communication between geographically dispersed sensors and actuators in order to obtain accurate state estimates. As with distributed control, there is a tradeoff between observer performance and the computational and communication load. One such approach based upon a distributed Kalman filter is presented, which allows for multi-rate sensor information to be utilized ${ }^{[104,105]}$. An attractive feature of this approach is that it converges to the optimal (centralized) solution of the state estimation problem. An integrated decentralized state feedback control and distributed state estimation procedure for linear and nonlinear systems is presented with a focus on parallel computation ${ }^{[106]}$. In addition to guaranteeing the stability and optimality of the observer (as well as the stability with the state feedback controller), the computational benefit of different decompositions of the process network (in terms of the number of distributed state estimators) is studied.

In [107], an approach which ensures a minimum $H_{\infty}$ 
performance bound on the state estimation over a finite time horizon is presented for time-varying systems with stochastic parameters and nonlinearities. To enable this framework, they developed a novel stochastic bounded real lemma to guarantee the $H_{\infty}$ performance. Also within the $H_{\infty}$ framework, a robust distributed state estimation was developed in [108], wherein observers with continuous time observations interact with one another at discrete time instances in a round-robin fashion. A cooperative distributed state estimation approach is presented in [109], wherein only communication between nearest neighbors is required to provide asymptotically stable state estimates in the presence of delays and dropouts in the estimator communication network.

As an analogue of MPC, moving horizon estimation (MHE), provides an alternative to (extended) Kalman filters for state estimation. Essentially, MHE finds the optimal state estimates over a finite rolling horizon of past input and output measurements subject to the process model constraints and constraints on the states and disturbances. With their ability to systematically handle state constraints, it has been argued that they are more applicable to chemical process systems than traditional Kalman filtering techniques ${ }^{[110-112]}$. As a consequence of the need for online optimization, MHE requires more computational effort than Kalman filters. However, in process industries, this may be not a major concern due to the relatively slow process dynamics, although it has been questioned whether the improved state estimations justify the increased computational effort ${ }^{[113]}$. The additional computational burden associated with MHE motivates distributed MHE approaches for large-scale systems.

Distributed MHE has gained interest in the literature recently in the same way that DMPC has. One practically appealing approach is the observer enhanced nonlinear distributed $\mathrm{MHE}^{[114,115]}$, whereby a known deterministic observer is utilized to provide a measurable level of robustness. This approach is somewhat similar to the Lyapunov DMPC approach discussed in Section 3.1.2, where a known controller is used to ensure the stability of the resulting closed-loop system. This approach has been extended to allow for event driven communication between observers ${ }^{[116]}$ to reduce the amount of required communication.

A distributed MHE approach with guaranteed convergence for linear networks was presented ${ }^{[117,118]}$. This has been extended to nonlinear networks ${ }^{[119,120]}$. The latter also allowed for the relaxation of the assumption of observability to detectability.

\section{Future directions of DPC}

Based on the review of the existing literature above, in this section, we discuss some possible areas for future research in the field of distributed process control. In particular, we discuss areas which can improve business efficiency.

\subsection{Flexible manufacturing and integra- tion with business objectives}

With increasing competition and changing industrial trends, more agile and flexible manufacturing methods are required for modern industrial processes ${ }^{[121,122]}$. An example of this is a shift in some areas away from steady state design and operation to semi-batch and batch designs, in order to decrease the time to market of different product lines and to allow for plant and equipment to be utilized for multiple product grades. The fast moving consumer goods industry is an example of such a case where this shift has occurred ${ }^{[123]}$. From a control perspective, this requires a shift away from traditional regulatory control of constant setpoints to allow for more frequent changes in product grade.

One approach to improve the flexibility and return on capital investment is reconfigurable control and process operations. The essential idea is to have set process units (reactors, dryers, filters, etc.) which can be connected together in different ways to efficiently produce different products or product grades. Examples of such cases are presented in the context of food processing ${ }^{[124]}$, for oil production $^{[125]}$, and for processing methane emissions ${ }^{[126]}$. A recent distributed approach to controlling such systems is presented $^{[122,127,128]}$, for which it is argued that distributed control structures are more suited to reconfigurable control than traditional hierarchical structures due to their increased flexibility.

A key motivation for flexible manufacturing is to make process operations more competitive by improving their agility, and to add value by providing mass customization of speciality products ${ }^{[121]}$. This is a significant step away from traditional continuous process operations which focused on steady state designs. However, there has been a recent trend towards flexible or "smart" plants ${ }^{[129,130]}$, in which processing operations are integrated with business needs and are made more agile allowing for swiftly moving business needs to be met. This latter point about smart plant operations is intimated related to distributed and networked control as the distribution of sensors, actuators and decision making is common to both.

An interesting line of research is to design "modular" distributed control systems which can be extended or grow if the plant changes, or individual local controllers can be updated with changing process equipment. This idea may be realized using the concepts of passivity/dissipativity or input to state stability (and the numerous variations of it in the literature), which allow for such a modular controller design. Developments in this area will allow for process networks to grow and expand without the need for a complete redesign of the control system every time a modification is made. This is related to flexible manufacturing and reconfigurable control as discussed above, as it facilitates the use (or retrofit) of installed equipment for multiple tasks.

Another important aspect of flexible manufacturing is the interaction with the product scheduling system, which 
chooses when and which products are to be produced. The product being produced is naturally a discrete, logical variable (i.e., product $\mathrm{A}, \mathrm{B}$ or $\mathrm{C}$ ), as such integration of this with process systems (which are continuous in nature) leads to a hybrid control problem, with a coupling of continuous dynamics and discrete events. Despite recent developments, this still remains a new area of research, and requires further work.

Along this note is the possibility for the control of operations to be integrated with a business' ${ }^{\prime}$ supply chain management scheme. This has the possibility of reducing the inventories that required, and allow for more effective scheduling of process operations. Furthermore, examples such as the MIT beer game suggest that supply chains can suffer from positive feedback leading to poor performance (i.e., oscillations or high gain) in a similar manner to which process networks have structures (i.e., mass recycle and heat integration) which may cause operability issues. This suggests that some of the DPC techniques may be used, or be adapted to be used in managing supply chains.

\subsection{Process monitoring and maintenance}

As process systems and their control systems become more complex, in order to achieve higher efficiency and tighter control performance, they become less transparent and harder to monitor and maintain. As such, there is a need to develop systems which can assist operators in monitoring and maintaining the plants control system. Distributed control structures are intrinsically well suited to this as they are more transparent than centralized control structures, and allow for a decomposition of the control system for analysis and maintenance.

Related to this is fault detection and isolation over (process) networks using sensor networks. This is an area of research which is still in its (relative) infancy, and as such should be considered as an area where significant gains, both theoretical and practical, can be achieved. Following this, the design of fault tolerant distributed control systems is of importance for their practical application in industry.

\subsection{Distributed economic model predic- tive control}

A recent development in the MPC literature is economic MPC, whereby the objective function in the online optimization measures some economic objective which is to be minimized ${ }^{[36,37,131]}$. The obvious advantage of this is that the optimization of the controller is then physically/economically motivated, as opposed to the mathematically convenient, but abstract quadratic cost functions often used in MPC. This may make the controller more transparent, as the controller is optimizing a tangible quantity. Distributed economic MPC is a natural extension to these developments, as in process networks, the economics of the overall system is intimately related to that of the individual processes and their interconnection relationships. Thus, distributed control is a powerful framework to deliver economic objectives in chemical process systems. Additionally, this may allow for a flattening of the plant-wide control structure, as the higher level economic optimization may be carried out at the lower levels of the control system.

Despite these advantages, there have been few developments in the area of distributed economic MPC in the literature. A difficulty with this approach is that for complicated systems (such as process networks), the economic cost function may be difficult to determine. Furthermore, the resulting optimization problem may be difficult to solve as the cost function may not lend itself to efficient optimization (e.g., it may not be convex) and the nonlinearity of the processes becomes much more important compared to regulating control where the setpoints are predetermined. However, due to advancements in computing power and optimization algorithms, these problems are being overcome, thus allowing for the benefits of distributed economic MPC to be realized.

\section{Conclusions}

A review of the current literature on distributed process control has been presented. A particular focus on distributed MPC has been taken due to its level of interest in the recent process control community, as well as its ability to handle nonlinearities and constraints which are key challenges in the control of chemical process networks. Whilst there has been significant innovations and developments in distributed process control, there are still outstanding practical issues, such as fault detection and tolerance, flexibility, reconfigurability and integration with scheduling and broader business objectives which need to be addressed to see a wider industrial implementation. Furthermore, integration of recently developed DPC technologies with discrete systems (such as PLCs or plant scheduling) to develop a hybrid control system would align research more closely with industrial practice.

Additionally, whilst loop pairing is not as important as in decentralized control schemes, it should be further developed for each local controller. Heuristics such as grouping variables in terms of the time-scale or based on geographic considerations, or from traditional chemical engineering tools (such as plant flow sheets) may be developed. This question is linked to the question of optimal controller communication network topology, which is still an open question in many cases.

Whilst this review has focused on distributed control in the context of process systems, there are other application areas where similar problems are encountered. For example, with a shift towards decentralized power generation due to renewable energy generation (e.g., rooftop solar panels) it is expected that greater strain will be placed on the electrical grid. Distributed control of generation and loads may provide a method of effectively maintaining stability of the electrical grid. Coordination problems such as in the control of platoons of vehicles or formations of air/spacecraft are also potential applications due to the importance of in- 
teractions between subsystems.

\section{References}

[1] A. M. Lenhoff, M. Morari. Design of resilient processing plants-I. Process design under consideration of dynamic aspects. Chemical Engineering Science, vol. 37, no. 2, pp. 245258, 1982.

[2] P. A. Bahri, J. A. Bandoni, J. A. Romagnoli. Integrated flexibility and controllability analysis in design of chemical processes. American Institute of Chemical Engineers Journal, vol. 43, no. 4, pp. 997-1015, 1997.

[3] J. D. Perkins, S. P. K. Walsh. Optimization as a tool for design/control integration. Computers and Chemical Engineering, vol. 20, no. 4, pp. 315-323, 1996.

[4] A. Kumar, P. Daoutidis. Nonlinear dynamics and control of process systems with recycle. Journal of Process Control, vol. 12 , no. 4, pp. 475-484, 2002.

[5] P. D. Christofides, R. Scattolini, D. Muñoz de la Peña, J. F. Liu. Distributed model predictive control: A tutorial review and future research directions. Computers and Chemical Engineering, vol. 51, pp. 21-41, 2013.

[6] S. Skogestad, M. Morari. Robust performance of decentralized control systems. Automatica, vol. 25, no. 1, pp.119$125,1989$.

[7] A. Swarnakar, H. J. Marquez, T. W. Chen. Multi-loop control synthesis for unstable systems and its application: An approach based on $\mu$ interaction measure. International Journal of Robust and Nonlinear Control, vol. 19, no. 15 , pp. 1721-1744, 2009 .

[8] S. Skogestad. Control structure design for complete chemical plants. Computers and Chemical Engineering, vol. 28, no. 1-2, pp. 219-234, 2004.

[9] Y. Ho. On centralized optimal control. IEEE Transactions on Automatic Control, vol. 50, no. 4, pp. 537-538, 2005.

[10] T. Larsson, S. Skogestad. Plantwide control-a review and a new design procedure. Modeling, Identification and Control, vol. 21, no. 4, pp. 209-240, 2000.

[11] J. F. Liu, X. Z. Chen, D. Muñoz de la Peña, P. D. Christofides. Sequential and iterative architectures for distributed model predictive control of nonlinear process systems. American Institute of Chemical Engineers Journal, vol. 56 , no. 8 , pp. $2137-2149,2010$.

[12] R. Scattolini. Architectures for distributed and hierarchical model predictive control-a review. Journal of Process Control, vol. 19, no. 5, pp. 723-731, 2009.

[13] W. L. Luyben, B. D. Tyréus, M. L. Luyben. Plantwide Process Control, New York, USA: McGraw-Hill, 1998.

[14] D. Hioe, J. Bao, B. E. Ydstie. Dissipativity analysis for networks of process systems. Computers and Chemical Engineering, vol. 50, pp. 207-219, 2013.
[15] W. L. Luyben. Snowball effects in reactor separator processes with recycle. Industrial \& Engineering Chemistry Research, vol. 33, no. 2, pp. 299-305, 1994

[16] S. Skogestad. Plantwide control: The search for the selfoptimizing control structure. Journal of Process Control, vol. 10, no. 5, pp. 487-507, 2000 .

[17] Y. Cao, Z. J. Yang. Multiobjective process controllability analysis. Computers and Chemical Engineering, vol. 28, no. 1-2, pp. 83-90, 2004.

[18] Y. Cao. Direct and indirect gradient control for static optimisation. International Journal of Automation and Computing, vol. 2, no. 1, pp.60-66, 2006.

[19] R. D'Andrea, G. E. Dullerud. Distributed control design for spatially interconnected systems. IEEE Transactions on Automatic Control, vol. 48, no. 9, pp. 1478-1495, 2003.

[20] N. Hudon, J. Bao. Dissipativity-based decentralized control of interconnected nonlinear chemical processes. Computers and Chemical Engineering, vol.45, pp. 84-101, 2012.

[21] B. T. Stewart, S. J. Wright, J. B. Rawlings. Cooperative distributed model predictive control for nonlinear systems. Journal of Process Control, vol. 21, no. 5, pp. 698-704, 2011.

[22] C. Langbort, V. Gupta. Minimal interconnection topology in distributed control design. SIAM Journal on Control and Optimization, vol.48, no. 1, pp. 397-413, 2009.

[23] C. Peng, Y. C. Tian, M. Tadé. State feedback controller design of networked control systems with interval time-varying delay and nonlinearity. International Journal of Robust and Nonlinear Control, vol. 18, no. 12, pp. 1285-1301, 2008.

[24] J. F. Liu, D. Muñoz de la Peña, B. J. Ohran, P. D. Christofides, J. F. Davis. A two-tier architecture for networked process control. Chemical Engineering Science, vol. 63 , no. 22 , pp. 5394-5409, 2008

[25] L. Acar. Boundaries of the receding horizon control for interconnected systems. Journal of Optimization Theory and Applications, vol. 84, no. 2, pp. 251-271, 1995.

[26] P. D. Christofides, J. F. Liu, D. Muñoz de la Peña. Networked and Distributed Predictive Control: Methods and Nonlinear Process Network Applications, New York, USA Springer, 2011.

[27] M. Farina, R. Scattolini. Distributed noncooperative MPC with neighbor-to-neighbor communication. In Proceedings of the 18th IFAC World Congress, IFAC, Milano, Italy, pp. 404-409, 2011.

[28] M. Farina, R. Scattolini. Distributed predictive control: A non-cooperative algorithm with neighbor-to-neighbor communication for linear systems. Automatica, vol. 48, no. 6 , pp. 1088-1096, 2012.

[29] G. Betti, M. Farina, R. Scattolini. Realization issues, tuning, and testing of a distributed predictive control algorithm. Journal of Process Control, vol. 24, no. 4, pp.424434,2014 
[30] W. B. Dunbar. Distributed receding horizon control of dynamically coupled nonlinear systems. IEEE Transactions on Automatic Control, vol. 52, no. 7, pp. 1249-1263, 2007.

[31] W. B. Dunbar, S. Desa. Distributed nonlinear model predictive control for dynamic supply chain management. In Proceedings of the International Workshop on Assessment and Future Directions on NMPC, Freudenstadt-Lauterbad, Germany, 2005.

[32] G. Y. Zhu, M. A. Henson. Model predictive control of interconnected linear and nonlinear processes. Industrial \& Engineering Chemistry Research, vol.41, no.4, pp.801-816, 2002.

[33] P. Mhaskar, N. H. El-Farra, P. D. Christofides. Predictive control of switched nonlinear systems with scheduled mode transitions. IEEE Transactions on Automatic Control, vol. 50, no. 11, pp. 1670-1680, 2005.

[34] P. Mhaskar, N. H. El-Farra, P. D. Christofides. Stabilization of nonlinear systems with state and control constraints using Lyapunov-based predictive control. Systems \& Control Letters, vol. 55, no. 8, pp. 650-659, 2006.

[35] M. Ellis, M. Heidarinejad, P. D. Christofides. Economic model predictive control of nonlinear two-time-scale systems. In Proceedings of the 21st Mediterranean Conference on Control and Automation, IEEE, Platanias-Chania, Crete, Greece, pp. 323-328, 2013.

[36] M. Ellis, J. Zhang, J. F. Liu, P. D. Christofides. Robust moving horizon estimation based output feedback economic model predictive control. System \& Control Letters, vol. 68, pp. 101-109, 2014.

[37] J. Zhang, S. Liu, J. F. Liu. Economic model predictive control with triggered evaluations: State and output feedback. Journal of Process Control, vol. 24, no. 8, pp.1197-1206, 2014.

[38] Y. J. Wang, J. B. Rawlings. A new robust model predictive control method I: Theory and computation. Journal of Process Control, vol. 14, no. 3, pp. 231-247, 2004.

[39] P. O. M. Scokaert, D. Q. Mayne. Min-max feedback model predictive control for constrained linear systems. IEEE Transactions on Automatic Control, vol. 43, no. 8, pp. 11361142,1998

[40] H. Genceli, M. Nikolaou. Robust stability analysis of constrained $l_{1}$-norm model predictive control. American Institute of Chemical Engineers Journal, vol.39, no. 12, pp. 1954-1965, 1993.

[41] D. Jia, B. H. Krogh. Min-max feedback model predictive control for distributed control with communication. In Proceedings of the American Control Conference, IEEE, Anchorage, USA, pp. 4507-4512, 2002.

[42] A. Richards, J. P. How. Robust distributed model predictive control. International Journal of Control, vol. 80, no. 9, pp. 1517-1531, 2007.

[43] D. Q. Mayne, J. B. Rawlings, C. V. Rao, P. O. M. Scokaert. Constrained predictive control: Stability and optimality. Automatica, vol. 36, no. 6, pp. 789-814, 2000.
[44] M. J. Tippett, J. Bao. Plant-wide dissipative model predictive control. AIChE Journal, vol. 59, no. 3 pp. 787-804, 2013.

[45] M. J. Tippett, J. Bao. A unified approach to plant-wide dissipative model predictive control. In Proceedings of the International Symposium on Advanced Control of Chemical Processes, IFAC, Singapore, pp. 420-425, 2012.

[46] T. Raff, C. Ebenbauer, P. Allgöwer. Nonlinear model predictive control: A passivity-based approach. In Proceedings of the International Workshop on Assessment and Future Directions of Nonlinear Model Predictive Control, Freudenstadt-Lauterbad, Germany, pp. 151-162, 2005.

[47] M. J. Tippett, J. Bao. Distributed dissipative model predictive control for process networks with imperfect communication. AIChE Journal, vol. 60, no. 5, pp. 1682-1699, 2014.

[48] C. X. Zheng, M. J. Tippett, J. Bao, J. F. Liu. Multirate dissipativity-based distributed MPC. In Proceedings of the 3rd Australian Control Conference, IEEE, Perth, Australia, pp. 325-330, 2013.

[49] C. Kojima, K. Takaba. A generalized Lyapunov stability theorem for discrete-time systems based on quadratic difference forms. In Proceedings of the 44th IEEE Conference on Decision and Control, and the European Control Conference, IEEE, Seville, Spain, pp. 2911-2916, 2005.

[50] C. Kojima, K. Takaba. An LMI condition for asymptotic stability of discrete-time system based on quadratic difference forms. In Proceedings of IEEE Conference on Computer Aided Control Systems Design, IEEE, Munich, Germany, pp. 1139-1143, 2006.

[51] J. B. Rawlings, B. T. Stewart. Coordinating multiple optimization-based controllers: New opportunities and challenges. Journal of Process Control, vol.18, no. 9, pp. 839-845, 2008.

[52] B. T. Stewart, A. N. Venkat, J. B. Rawlings, S. J. Wright, G. Pannocchia. Cooperative distributed model predictive control. Systems \& Control Letters, vol. 59, no. 8, pp.460469, 2010.

[53] A. N. Venkat, J. B. Rawlings, S. J. Wright. Stability and optimality of distributed model predictive control. In Proceedings of the 44th IEEE Conference on Decision and Control, and the European Control Conference, IEEE, Seville, Spain, pp. 6680-6685, 2005.

[54] A. N. Venkat, J. B. Rawlings, S. J. Wright. Implementable distributed model predictive control with guaranteed performance properties. In Proceedings of the American Control Conference, IEEE, Minneapolis, USA, 2006.

[55] P. O. M. Scokaert, D. Q. Mayne, J. B. Rawlings. Suboptimal model predictive control (feasibility implies stability). IEEE Transactions on Automatic Control, vol.44, no. 3, pp. 648-654, 1999.

[56] A. Ferramosca, D. Limon, I. Alvarado, E. F. Camacho. Cooperative distributed MPC for tracking. Automatica, vol. 49, no. 4, pp. 906-914, 2013. 
[57] H. Scheu, J. Busch, W. Marquardt. Nonlinear distributed dynamic optimization based on first order sensitivities. In Proceedings of the American Control Conference, IEEE, Baltimore, USA, pp. 1574-1579, 2010.

[58] H. Scheu, W. Marquardt. Sensitivity-based coordination in distributed model predictive control. Journal of Process Control, vol. 21, no. 5, pp. 715-728, 2011.

[59] J. M. Maestre, D. Muñoz de la Peña, E. F. Camacho. Distributed model predictive control based on a cooperative game. Optimal Control Applications and Methods, vol. 32, no. 2, pp. 153-176, 2011

[60] J. M. Maestre, D. Muñoz de la Peña, E. F. Camacho, T. Alamo. Distributed model predictive control based on agent negotiation. Journal of Process Control, vol. 21, no. 5 , pp. 685-697, 2011.

[61] J. F. Liu, D. Muñoz de la Peña, P. D. Christofides. Distributed model predictive control of nonlinear process systems. American Institute of Chemical Engineers Journal, vol. 55, no. 5, pp. 1171-1184, 2009.

[62] J. F. Liu, D. Muñoz de la Peña, P. D. Christofides. Distributed model predictive control of nonlinear systems subject to asynchronous and delayed measurements. Automatica, vol. 46, no. 1, pp. 52-61, 2010.

[63] M. Heidarinejad, J. F. Liu, D. Muñoz de la Peña, J. F. Davis, P. D. Christofides. Multirate Lyapunovbased distributed model predictive control of nonlinear uncertain systems. Journal of Process Control, vol. 21, no. 9, pp. 1231$1242,2011$.

[64] X. Z. Chen, M. Heidarinejad, J. F. Liu, D. Muñoz de la Peña, P. D. Christofides. Model predictive control of nonlinear singularly perturbed systems: Application to a largescale process network. Journal of Process Control, vol. 21, no. 9, pp. 1296-1305, 2011.

[65] M. Heidarinejad, J. F. Liu, D. Muñoz de la Peña, J. F. Davis, P. D. Christofides. Handling communication disruptions in distributed model predictive control. Journal of Process Control, vol. 21, no. 1, pp. 173-181, 2011.

[66] X. Z. Chen, M. Heidarinejad, J. F. Liu, P. D. Christofides. Distributed economic MPC: Application to a nonlinear chemical process network. Journal of Process Control, vol. 22, no. 4, pp. 689-699, 2012.

[67] R. M. Hermans, A. Jokić, M. Lazar, A. Alessio, P. P. J. van den Bosch, I. A. Hiskens, A. Bemporad. Assessment of non-centralised model predictive control techniques for electrical power networks. International Journal of Control, vol. 85, no. 8, pp. 1162-1177, 2012.

[68] E. Camponogara, H. F. Scherer. Distributed optimization for model predictive control of linear dynamic networks with Controlinput and output constraints. IEEE Transactions on Automation Science and Engineering, vol. 8, no. 1, pp. 233-242, 2011.

[69] I. Necoara, V. Nedelcu, I. Dumitrache. Parallel and distributed optimization methods for estimation and control in networks. Journal of Process Control, vol. 21, no. 5, pp. 756766,2011
[70] Y. Zheng, S. Y. Li, N. Li. Distributed model predictive control over network information exchange for large-scale systems. Control Engineering Practice, vol. 19, no. 7, pp. 757$769,2011$.

[71] X. Cai, M. J. Tippett, L. Xie, J. Bao. Fast distributed MPC based on active set method. Computers and Chemical Engineering, vol. 71, pp. 158-170, 2014.

[72] P. Giselsson, M. D. Doan, T. Keviczky, B. de Schutter, A. Rantzer. Accelerated gradient methods and dual decomposition in distributed model predictive control. Automatica, vol. 49, no. 3, pp. 829-833, 2013.

[73] I. Necoara, D. Doan, J. A. K. Suykens. Application of the proximal center decomposition method to distributed model predictive control. In Proceedings of the 47th IEEE conference on Decision and Control, IEEE, Cancun, Mexico, pp. 2900-2905, 2008 .

[74] S. D. Cairano, M. Brand, S. A. Bortoff. Projection-free parallel quadratic programming for linear model predictive control. International Journal of Control, vol. 86, no. 8 , pp. 1367-1385, 2013.

[75] A. Bemporad, M. Morari, V. Dua, E. N. Pistikopoulos. The explicit linear quadratic regulator for constrained systems. Automatica, vol. 38, no. 1, pp. 3-20, 2002.

[76] T. A. Johansson, I. Petersen, O. Slupphaug. Explicit Suboptimal linear quadratic regulation with state and input constraints. Automatica, vol. 38, no. 7, pp. 1099-1111, 2002.

[77] J. C. Willems. Dissipative dynamical systems, Part I: General theory. Archive for Rational Mechanics and Analysis, vol. 45, no. 5, pp. 321-351, 1972.

[78] J. C. Willems. Dissipative dynamical systems, Part II: Linear systems with quadratic supply rates. Archive for Rational Mechanics and Analysis, vol.45, no. 5, pp. 352-393, 1972.

[79] D. J. Hill, P. Moylan. The stability of nonlinear dissipative systems. IEEE Transactions on Automatic Control, vol. 21 no. 5, pp. 708-711, 1976.

[80] D. J. Hill, P. J. Moylan. Stability results for nonlinear feedback systems. Automatica, vol. 13, no. 4, pp. 377-382, 1977.

[81] P. J. Moylan, D. J. Hill. Stability criteria for large-scale systems. IEEE Transactions on Automatic Control, vol. 23, no. 2 pp. $143-149,1978$.

[82] A. A. Alonso, B. E. Ydstie. Process systems, passivity and the second law of thermodynamics. Computers and Chemical Engineering, vol. 20, no. S2, pp. S1119-S1124, 1996.

[83] A. A. Alonso, B. E. Ydstie. Stabilization of distributed systems using irreversible thermodynamics. Automatica, vol. 37, no. 11, pp. 1739-1755, 2001.

[84] K. M. Hangos, A. A. Alonso, J. D. Perkins, B. E. Ydstie. Thermodynamic approach to the structural stability of process plants. American Institute of Chemical Engineers Journal, vol. 45, no. 4, pp. 802-816, 1999. 
[85] A. A. Alonso, C. V. Fernandez, J. R. Banga. Dissipative systems: From physics to robust 2nonlinear control. International Journal of Robust and Nonlinear Control, vol. 14, no. 2, pp. 157-179, 2004.

[86] M. Baldea, N. H. El-Farra, B. E. Ydstie. Dynamics and control of chemical process networks: Integrating physics, communication and computation. Computers and Chemical Engineering, vol. 51, pp. 42-54, 2013.

[87] B. E. Ydstie, A. A. Alonso. Process systems and passivity via the Clausius-Planck inequality. Systems \& Control Letters, vol. 30, no. 5, pp. 253-264, 1997.

[88] K. R. Jillson, B. E. Ydstie. Process networks with decentralized inventory and flow control. Journal of Process Control, vol. 17, no. 5, pp. 399-413, 2007.

[89] R. Setiawan, J. Bao. Analysis of interaction effects on plantwide operability. Industrial \& Engineering Chemistry Research, vol. 50, no. 14, pp. 8585-8602, 2011.

[90] R. Setiawan, J. Bao. Plantwide operability assessment for nonlinear processes using a microscopic level network analysis. Chemical Engineering Research and Design, vol. 90, no. 1, pp. 119-128, 2012.

[91] O. J. Rojas, R. Setiawan, J. Bao, P. L. Lee. Dynamic operability analysis of nonlinear process networks based on dissipativity. AIChE Journal, vol. 55, no. 4, pp. 963-982, 2009.

[92] G. Scorletti, G. Duc. A convex approach to decentralized $H_{\infty}$ control. In Proceedings of the American Control Conference, IEEE, Albuquerque, New Mexico, pp. 2390-2394, 1997.

[93] G. Scorletti, G. Duc. An LMI approach to decentralized $H_{\infty}$ control. International Journal of Control, vol. 74 , no. 3 pp. 211-224, 2001.

[94] S. C. Xu, J. Bao. Distributed control of plantwide chemical processes. Journal of Process Control, vol. 19, no. 10, pp. 1671-1687, 2009.

[95] S. C. Xu, J. Bao. Control of chemical processes via output feedback controller networks. Industrial \& Engineering Chemistry Research, vol. 49, no. 16, pp. 7421-7445, 2010.

[96] M. J. Tippett, J. Bao. Control of plant-wide systems using dynamic supply rates. Automatica, vol. 50, no. 1, pp. 44-52, 2014.

[97] M. J. Tippett, J. Bao. Dissipativity based distributed control synthesis. Journal of Process Control, vol. 23, no. 5, pp. 755-766, 2013.

[98] C. Langbort, R. S. Chandra, R. D'Andrea. Distributed control design for systems interconnected over an arbitrary graph. IEEE Transactions on Automatic Control, vol. 49, no. 9, pp. 1502-1519, 2004.

[99] C. Schizas, F. J. Evans. A graph theoretic approach to multivariable control system design. Automatica, vol. 17, no. 2, pp. 371-377, 1981.
[100] M. Vidyasagar. Input-output Analysis of Large-scale Interconnected Systems, Berlin, Grermany: Springer-Verlag, 1981.

[101] C. Langbort, V. Gupta. Minimal interconnection topology in distributed control design. In Proceedings of the American Control Conference, IEEE, Minnepolis, USA, pp. 845850, 2006.

[102] K. M. Hangos, Z. Tuza. Optimal control structure selection for process systems. Computers \& Chemical Engineering, vol. 25, no. 11-12, pp. 1521-1536, 2001.

[103] P. Lin, Y. M. Jia, L. Li. Distributed robust $H_{\infty}$ consensus control in directed networks of agents with time-delay. Systems \& Control Letters, vol. 57, no. 8, pp. 643-653, 2008.

[104] R. Vadigepalli, F. J. Doyle. A distributed state estimation and control algorithm for plantwide processes. IEEE Transactions on Control Systems Technology, vol. 11, no. 1, pp. 119-127, 2003.

[105] R. Vadigepalli, F. J. Doyle. Structural analysis of largescale systems for distributed state estimation and control applications. Control Engineering Practice, vol.11, no. 8, pp. 895-905, 2003.

[106] N. Abdel-Jabbar, C. Kravaris, B. Carnahan. A partially decentralized state observer and its parallel computer implementation. Industrial \& Engineering Chemistry Research, vol.37, no. 7, pp. 2741-2760, 1998.

[107] D. R. Ding, Z. D. Wang, H. L. Dong, H. S. Shu. Distributed H1 state estimation with stochastic parameters and nonlinearities through sensor networks: The finite horizon case. Automatica, vol. 48, no. 8, pp. 1575-1585, 2012.

[108] V. Ugrinovskii, E. Fridman. A round-robin type protocol for distributed estimation with $H_{\infty}$ consensus. Systems \& Control Letters, vol. 69, pp. 103-110, 2014.

[109] P. Millán, L. Orihuela, C. Vivas, F. R. Rubio. Distributed consensus-based estimation considering network induced delays and dropouts. Automatica, vol. 48, no. 10, pp. $2726-$ $2729,2012$.

[110] E. L. Haseltine, J. B. Rawlings. Critical evaluation of extended kalman filtering and moving-horizon estimation. Industrial \& Engineering Chemistry Research, vol. 44, no. 8, pp. 2451-2460, 2005.

[111] C. V. Rao, J. B. Rawlings, J. H. Lee. Constrained linear state estimation-a moving horizon approach. Automatica, vol. 37, no. 10, pp. 1619-1628, 2001.

[112] C. V. Rao, J. B. Rawlings, D. Q. Mayne. Constrained state estimation for nonlinear discrete-time systems: Stability and moving horizon approximations. IEEE Transactions on Automatic Control, vol. 48, no. 2, pp. 246-258, 2003.

[113] J. Busch, D. Elixmann, P. Kühl, C. Gerkens, J. P. Schöder, H. G. Bock, W. Marquardt. State estimation for largescale wastewater treatment plants. Water Research, vol. 47, no. 13 , pp. $4774-4787,2013$. 
[114] J. F. Liu. Moving horizon state estimation for nonlinear systems with bounded uncertainties. Chemical Engineering Science, vol. 93, pp. 376-386, 2013.

[115] J. Zhang, J. F. Liu. Observer-enhanced distributed moving horizon state estimation subject to communication delays. Journal of Process Control, vol. 24, no. 5, pp. 672-686, 2014.

[116] J. Zhang, J. F. Liu. Two triggered information transmission algorithms for distributed moving horizon state estimation. Systems \& Control Letters, vol. 65, pp. 1-12, 2014.

[117] M. Farina, G. Ferrari-Trecate, R. Scattolini. Distributed moving horizon estimation for linear constrained systems. IEEE Transactions on Automatic Control, vol. 55, no. 11, pp. $2462-2475,2010$.

[118] M. Farina, G. Ferrari-Trecate, R. Scattolini. Movinghorizon partition-based state estimation of large-scale systems. Automatica, vol. 46, no. 5, pp. 910-918, 2010.

[119] M. Farina, G. Ferrari-Trecate, C. Romani, R. Scattolini. Moving horizon estimation for distributed nonlinear systems with application to cascade river reaches. Journal of Process Control, vol. 21, no. 5, pp. 767-774, 2011.

[120] M. Farina, G. Ferrari-Trecate, R. Scattolini. Distributed moving horizon estimation for nonlinear constrained systems. International Journal of Robust and Nonlinear Control, vol. 22, no. 2, pp. 123-143, 2012.

[121] N. Shah. Process industry supply chains: Advances and challenges. Computers \& Chemical Engineering, vol. 29, no. 6, pp. 1225-1235, 2005.

[122] N. N. Chokshi, D. C. McFarlane. A Distributed Coordination Approach to Reconfigurable Process Control, London: Springer-Verlag, 2008.

[123] G. E. Keller, P. F. Bryan. Process engineering: Moving in new directions. Chemical Engineering Progress, vol. 96, no. 1 , pp. 41-50, 2000

124] T. Wauters, K. Verbeeck, P. Verstraete, G. Vanden Berghe, P. De Causmaecker. Real-world production scheduling for the food industry: An integrated approach. Engineering Applications of Artificial Intelligence, vol. 25, no. 2, pp. 222228,2012

[125] E. Chacón, I. Besembel, J. C. Hennet. Coordination and optimization in oil and gas production complexes. Computers in Industry, vol. 53, no. 1, pp. 17-37, 2004.

[126] S. Salomons, R. E. Hayes, M. Poirier, H. Sapoundjiev. Modelling a reverse flow reactor for the catalytic combustion of fugitive methane emissions. Computers \& Chemical Engineering, vol. 28, no. 9, pp. 1599-1610, 2004.
[127] N. Chokshi, D. C. McFarlane. A distributed architecture for reconfigurable control of continuous process operations. Journal of Intelligent Manufacturing, vol. 19, no. 2, pp. 215232,2008

[128] M. J. Tippett, J. Bao. Reconfigurable distributed model predictive control. Chemical Engineering Science, [Online], Available: $\quad$ http://dx.doi.org/10.1016/j.ces.2015.01.040, 2015.

[129] P. Christofides, J. Davis, N. H. El-Farra, D. Clark, H. K. K. R. D. Harris, J. N. Gipson. Smart plant operations: Vision, progress and challenges. American Institute of Chemical Engineers Journal, vol. 53, no. 11, pp. 2734-2741, 2007.

[130] B. Erik Ydstie. New vistas for process control: Integrating physics and communication networks. AIChE Journal, vol. 48 , no. 3 , pp. 422-426, 2002 .

[131] D. Angeli, R. Amrit, J. B. Rawlings. On average performance and stability of economic model predictive control. IEEE Transactions on Automatic Control, vol.57, no. 7, pp. 1615-1626, 2012.

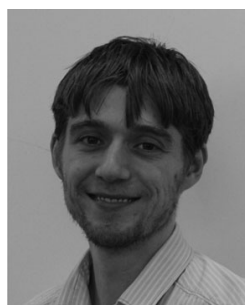

Michael J. Tippett received the B. Eng. degree (1st Class Honours and University Medal) in industrial chemistry from The University of New South Wales (UNSW), Australia in 2009, and the $\mathrm{Ph} . \mathrm{D}$. degree from the same university in 2014. He has held a postdoctoral fellowship at UNSW and is currently an engineer at OSIsoft LLC and an adjunct lecturer in Chemical Engi-

neering at UNSW.

His research interests include distributed control and decision making for plant-wide process control, dissipativity based control, distributed model predictive control, and fault detection and the design of fault tolerant control systems.

E-mail: michael. tippett@unsw.edu.cn

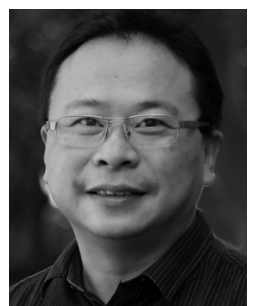

Jie Bao received the B. Sc. the and M. Sc degrees in electrical engineering from Zhejiang University, China in 1990 and 1993 respectively. In 1998, he received the $\mathrm{Ph}$. D degree in chemical engineering (process control) from The University of Queensland, Australia. He spent one year at University of Alberta as a postdoctoral fellow and then joined the faculty at The University of New South Wales, Australia. He is currently a full professor in the School of Chemical Engineering, UNSW. He is an associate editor of Journal of Process Control.

His research interests include distributed and decentralized control, robust control, fault-tolerant control, dissipativity-based process control and control of industrial processes including aluminium smelting, mineral processing, membrane separation and flow batteries.

E-mail: j.bao@unsw.edu.au (Corresponding author)

ORCID iD: 0000-0002-6774-9863 